\title{
COUNTERPOINT: Aortic valve replacement: Size does matter
}

\author{
Christopher M. Feindel, MD
}

Patients with aortic stenosis are rarely denied aortic valve replacement today, and with successful surgery they can expect excellent symptomatic relief and improved life expectancy. However, after Shabudin Rahimtoola's ${ }^{1}$ observation in 1978 that a prosthetic valve may not fully relieve a patient's aortic stenosis, a condition he referred to as "valve prosthesis-patient mismatch" (PPM), surgeons became wary of the potential harm of inserting small valve prostheses. ${ }^{1}$ Rahimtoola's concerns were further supported by the Framingham Study, as well as others, which showed that patients with left ventricular hypertrophy had a reduced life expectancy compared with those without left ventricular hypertrophy. ${ }^{2,3}$ Because left ventricular hypertrophy does not regress in patients with severe valve PPM, the natural conclusion was that patients with PPM fare worse than those who do not have mismatch. A number of studies have confirmed this to be the case, at least in the early postoperative period, and particularly in those patients with poor ventricular function. ${ }^{4,5}$ Surgeons realized the risk of valve PPM could be minimized if valves were implanted that provided the patient with an effective valve orifice area of greater than $0.85 \mathrm{~cm}^{2} / \mathrm{m}^{2}$. Since all mechanical and stented bioprostheses are to some degree obstructive due to the presence of sewing rings and stents, this is not always possible, especially in small patients. Tasca and colleagues ${ }^{6}$ showed that more than $55 \%$ of patients in whom a $21-\mathrm{mm}$ bioprosthetic valve was inserted had either moderate or severe PPM, and this figure increased to more than $90 \%$ in those who had a $19-\mathrm{mm}$ valve inserted.

Over time, a number of surgical techniques were developed to allow the insertion of appropriately sized prostheses, techniques described by Konno and colleagues, ${ }^{7}$ Nicks and colleagues, ${ }^{8}$ and Manouguian and Seybold-Epting. ${ }^{9}$ Although these techniques worked well, they also added to the complexity of the surgery, especially in Konno and colleagues' procedure. This added complexity may have contributed to a higher early perioperative mortality. ${ }^{10}$ Although this increased complexity and possible increased mortality discouraged some surgeons from performing aor-

From the Division of Cardiovascular Surgery, Peter Munk Cardiovascular Centre, Toronto General Hospital and the University of Toronto, Toronto, Ontario, Canada. Presented at the 88th Annual Meeting of the American Association for Thoracic Surgery by Dr. Marc Moon.

Received for publication June 1, 2008; accepted for publication Aug 31, 2008.

Address for reprints: Christopher M. Feindel, MD, Division of Cardiovascular Surgery, Peter Munk Cardiovascular Centre, Toronto General Hospital and the University of Toronto, 200 Elizabeth St, 4N-480, Toronto, Ontario, Canada M5G 2C4 (E-mail: chris.Feindel@uhn.on.ca).

J Thorac Cardiovasc Surg 2009;137:284-5

$0022-5223 / \$ 36.00$

Copyright (c) 2009 by The American Association for Thoracic Surgery doi:10.1016/j.jtcvs.2008.08.059 tic root enlargement, more recent series have shown that in experienced hands aortic root enlargement does not increase perioperative risk. Although there seems to be an early survival benefit of avoiding PPM in these studies, the long-term benefit of avoiding mismatch seems less clear. $^{11-13}$

In this series, reported by Dr Moon, 1399 patients who underwent bioprosthetic AVR between 1992 and 2007 by 22 different surgeons were evaluated for early and late mortality and the presence or absence of PPM. By using a "Social Security Death Index" to track long-term mortality, Dr Moon observed that in patients aged less than 70 years the presence of PPM was associated with impaired long-term survival. However, this was not the case in patients aged more than 70 years. On the basis of these observations, he concluded that "PPM had a negative impact on late survival for patients less than or equal to 70 years of age, but for patients greater than 70 years of age, PPM did not influence survival.' Although not explicitly stated in this study, the obvious implication to surgeons is that aortic root enlargement techniques are not necessary in elderly patients, especially if they think they may be exposing their patients to an added risk during aortic valve replacement.

Although Dr Moon observed that there was an association between mortality and PPM, his analysis does not provide sufficient information to define what that impact may be. The main reason for this is his use of the Death Index Registry to identify mortality in this series. There was no reported direct patient follow-up, which means that there is no way of knowing the cause of death in any of the patients. As a result, one cannot make any inference about the impact of PPM on long-term survival in any of the groups. Following Dr Moon's methodology, it seems that in the older patients, 10-year survival was $42 \% \pm 6 \%$ in those with severe PPM compared with $29 \% \pm 5 \%$ in those without PPM, suggesting the unusual conclusion that severe PPM actually conferred a survival benefit to the older patients! This is highly unlikely to be the case and simply emphasizes the inherent weakness of attempting to attach causation to an isolated observation (in this case PPM) without identifying the actual cause of death. Although Dr Moon makes some interesting observations in this study, the reader must be cautioned not to come to any conclusions about the influence that PPM may or may not have on survival in any of the groups studied in this series.

As cardiac surgeons, what should we do for the patient with a small aortic annulus? Although most of us believe that PPM is harmful to patients, we are unlikely to ever see a randomized trial determining whether mismatch reduces long-term survival. Consequently, we are left with using indirect evidence to guide our practices. Because 
patients with left ventricular hypertrophy do worse than those without, and PPM after aortic valve replacement results in persistent left ventricular hypertrophy, most agree that mismatch is not good for our patients. At the Toronto General Hospital we discourage the use of small valves, especially $19-\mathrm{mm}$ bioprosthetic valves. Whenever possible, $23-\mathrm{mm}$ tissue valves for women and $25-\mathrm{mm}$ tissue valves for men are used, although $21 \mathrm{~mm}$ for women and $23 \mathrm{~mm}$ for men are acceptable for elderly inactive individuals. To adhere to these guidelines, aortic root enlargement is used more than $75 \%$ of the time in my own practice.

Aortic root enlargement, whether Nicks and colleagues' or Manouguian and Seybold-Epting's procedure, is a valuable adjunct for the practicing cardiac surgeon, provided it is performed well. By adhering to certain details, aortic root enlargement can be performed safely and does not appreciably add to the complexity of the surgery. One cannot overemphasize that it is the meticulous attention to details that will ensure the success of the procedure. I favor Nicks and colleagues' procedure, which extends the aortotomy in the noncoronary sinus across the mitral annulus for no more than 1 to $1.5 \mathrm{~cm}$. Untreated autologous pericardium ( $\sim 5$ to 7 -cm oval patch) is used to reconstruct this area using a double suture layer (first a 4-0 followed by a 5-0 polypropylene suture), which guarantees hemostasis in the critical area that will be below the valve sewing ring. Pledgeted valve sutures are placed around the annulus, and in the region of the patch these pledgets should be placed on the outside of the patch. In this area one should use large pledgets to avoid tearing through the patch. ${ }^{14}$

There is an added advantage to the frequent use of aortic root enlargement techniques. How often, after removal of a heavily calcified aortic valve, are we left with a damaged aortic annulus in which it will be difficult to implant a valve prosthesis? How often do we encounter difficulties in safely closing the aortic wall of a rigid calcified aortic in a frail elderly person? Surgeons facile with the use of pericardial patches, whether bovine or autologous, to enlarge the aortic root are usually very comfortable with reconstruction techniques required to manage these unexpected yet not uncommon situations.

No doubt some surgeons will continue to advocate that a safely performed aortic valve replacement using a small valve is better than adding the potential risk of an aortic route enlargement procedure. However, they must also accept that these patients will almost certainly leave the operating room with PPM along with its potential harmful consequences.
In the end, it will be up to the individual surgeon to balance the risk of performing an aortic root enlargement procedure with the risk of PPM. My own view is that aortic root enlargement is a reproducible procedure that can safely be performed by any surgeon performing aortic valve surgery. Young trainees are eager to learn aortic root enlargement techniques, and those who are comfortable with routine aortic valve replacement should have no difficulty in performing them under supervision. In our University of Toronto Cardiac Surgery Residency Program we make every effort to ensure that trainees are provided with such opportunities by mentors who are comfortable with aortic root enlargement techniques.

Dr Moon's research, although interesting, has not convinced me that we can ignore PPM in any patient group regardless of age. In my opinion, size indeed does matter!

\section{References}

1. Rahimtoola SH. The problem of valve prosthesis-patient mismatch. Circulation 1978;58:20-4.

2. Kannel WB, Levy D, Cupples LA. Left ventricular hypertrophy and risks of cardiac failure: insights from the Framingham Study. J Cardiovasc Pharmacol. 1987;10(Suppl 6):S135-40.

3. Wachthell K, Okin P, Olsen M, et al. Regression of electrocardiographic left ventricular hypertrophy during antihypertensive therapy and reduction in sudden cardiac death. Circulation. 2007;116:700-5.

4. Dumesnil JG, Phibarot P. Prosthesis-patient mismatch and clinical outcomes: the evidence continues to accumulate. J Thorac Cardiovasc Surg. 2006;131: 952-5.

5. Blais C, Dumesnil JG, Baillot R, Simard S, Doyle D, Pibarot P. Impact of valve prosthesis-patients mismatch on short-term mortality after aortic valve replacement. Circulation. 2003;108:983-8.

6. Tasca G, Brunelli F, Cirillo M, et al. Impact of valve prosthesis-patient mismatch on left ventricular mass regression following aortic valve replacement. Ann Thorac Surg. 2005;79:505-10.

7. Konno S, Imai Y, Iida Y, Nakajima M, Tatsuno K. A new method for prosthetic valve replacement in congenital aortic stenosis associated with hypoplasia of the aortic ring. J Thorac Cardiovasc Surg. 1975;70:909-17.

8. Nicks R, Cartmill T, Bernstein L. Hypoplasia of the aortic root. Thorax. 1970;25: 339-46.

9. Manouguian S, Seybold-Epting W. Patch enlargement of the aortic valve ring by extending the aortic incision into the anterior mitral leaflet. J Thorac Cardiovasc Surg. 1979;78:402-12.

10. Sommers KE, David TE. Aortic valve replacement with patch enlargement of the aortic annulus. Ann Thorac Surg. 1997;63:1608-12.

11. Kulik A, Al-Saigh M, Chan V, et al. Enlargement of the small aortic root during aortic valve replacement: is there a benefit? Ann Thorac Surg. 2008;85:94-101.

12. Rao V, Jamieson E, Ivanov J, Armstrong S, David TE. Prosthesis-patien mismatch affects survival after aortic valve replacement. Circulation. 2000; 102(suppl III):III-5-9.

13. Peterson MD, Borger MA, Feindel CM, David TE. Aortic annular enlargement during aortic valve replacement: improving results with time. Ann Thorac Surg. 2007;83:2044-9.

14. Feindel CM. Aortic root enlargement in the adult. Operative techniques in thoracic and cardiovascular surgery: a comparative atlas. In: Cohn LH, ed. New York: Elsevier; 2006. p. 2-15 